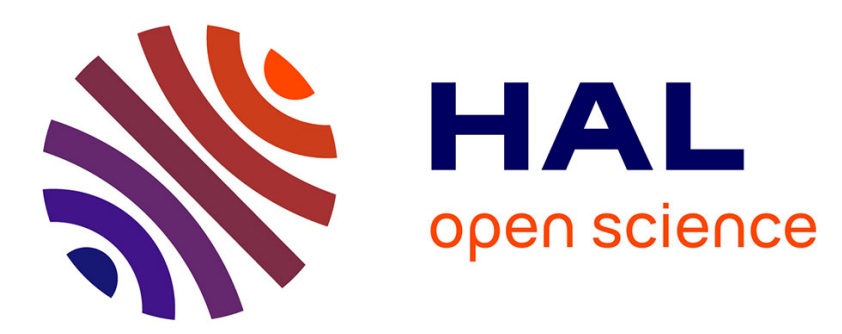

\title{
The influence of spherical cavity surface charge distribution on the sequence of partial discharge events
}

\author{
Hazlee A Illias, George Chen, Paul L Lewin
}

\section{To cite this version:}

Hazlee A Illias, George Chen, Paul L Lewin. The influence of spherical cavity surface charge distribution on the sequence of partial discharge events. Journal of Physics D: Applied Physics, 2011, 44 (24), pp.245202. 10.1088/0022-3727/44/24/245202 . hal-00625564

\section{HAL Id: hal-00625564 https://hal.science/hal-00625564}

Submitted on 22 Sep 2011

HAL is a multi-disciplinary open access archive for the deposit and dissemination of scientific research documents, whether they are published or not. The documents may come from teaching and research institutions in France or abroad, or from public or private research centers.
L'archive ouverte pluridisciplinaire HAL, est destinée au dépôt et à la diffusion de documents scientifiques de niveau recherche, publiés ou non, émanant des établissements d'enseignement et de recherche français ou étrangers, des laboratoires publics ou privés. 


\title{
The influence of spherical cavity surface charge distribution on the sequence of partial discharge events
}

\author{
Hazlee A. Illias, George Chen, Paul L. Lewin \\ The Tony Davies High Voltage Laboratory, School of Electronics and Computer \\ Science, University of Southampton, Southampton, SO17 1BJ United Kingdom \\ E-mail: hai07r@ecs.soton.ac.uk
}

\begin{abstract}
In this work, a model representing partial discharge (PD) behaviour of a spherical cavity within a homogeneous dielectric material has been developed to study the influence of cavity surface charge distribution on the electric field distribution in both the cavity and the material itself. The charge accumulation on the cavity surface after a PD event and charge movement along the cavity wall under the influence of electric field magnitude and direction has been found to affect the electric field distribution in the whole cavity and in the material. This in turn affects the likelihood of any subsequent PD activity in the cavity and the whole sequence of PD events. The model parameters influencing cavity surface charge distribution can be readily identified; they are the cavity surface conductivity, the inception field and the extinction field. Comparison of measurement and simulation results has been undertaken to validate the model.
\end{abstract}




\section{Introduction}

Partial discharge (PD) monitoring has become an essential tool in assessing the performance of insulation systems, consequently the modelling of PD is important in providing a better understanding of the phenomena. Although PD does not cause direct breakdown of the insulation, it indicates the presence of defects within the insulation, which may cause energy loss and insulation degradation. PD activity in a dielectric-bounded cavity is influenced by many factors. One of them is the surface charge distribution along the cavity wall, which modifies the electric field distribution in the cavity and influences the time of the next discharge occurrence [1, 2]. Previous PD models have effectively reproduced PD patterns of measurements data [3]- [5]. However, the distribution of surface charge along the cavity wall due to PD events is not detailed by these models, where the whole electric field distribution in the cavity is assumed to be uniform. Although surface charge distribution in a dielectric-bounded cavity cannot be directly measured, it is possible to incorporate its effects into a model [6, 7]. A model of PD in a dielectric-bounded cylindrical cavity has been developed by including the effect of charge distribution left on the cavity surface on subsequent discharges [8]. The effect of surface conductivity and discharge area on surface charge distribution in voids have also been investigated [9].

In this work, a model representing PD behaviour within a spherical cavity in a homogeneous dielectric material has been developed using the Finite Element Analysis (FEA) method. The model has been used to study the influence of cavity surface charge distribution on the electric field distribution in the cavity and the material. The cavity surface charge distribution is determined by charge propagation on the cavity surface during a PD event and charge movement along the cavity wall under the influence of electric field. The model parameters influencing surface charge distribution can also be identified; these include the cavity surface conductivity, inception field and extinction

field. Comparison of measurement and simulation results has been undertaken to validate the simulation results.

\section{2. $P D$ in a cavity}

A cavity is a gas-filled void within a dielectric material which may be created during manufacture, installation or during operation of a high voltage system [10]. To ensure PD activity occurs in a cavity, the electric field in the cavity must exceed the breakdown strength of the gas, which is called the inception field, $E_{\text {inc }}$ and there must also be a free electron available to initiate an electron avalanche [11].

A generalized PD event in a cavity is illustrated in figure $1[4,12]$. With reference to figure 1(a), all fields have the same polarity as the applied voltage. Before a discharge occurs, the electric field in the cavity centre, $E_{c a v}$ is equal to $f_{c} E_{0}$ where $f_{c}$ is the modification factor of the applied field [3], $E_{0}$ in the cavity, depending on the cavity geometry and the material permittivity (figure 1(a)). During a PD, once the discharge 
has passed through the cavity to the opposite surface, charges propagate along the cavity wall (figure $1(\mathrm{~b})$ ). This causes $E_{\text {cav }}$ to decrease because a field in the opposite direction, $E_{s}$ is developed (figure $1(\mathrm{c})$ ). Thus, $E_{c a v}$ is reduced and $E_{c a v}$ is the vector sum of $f_{c} E_{0}$ and $E_{s}$. When this happens, free electrons lose their energy and the current in the discharge channel decreases. When the field in the cavity drops to less than the extinction field, $E_{\text {ext }}$ discharge stops.

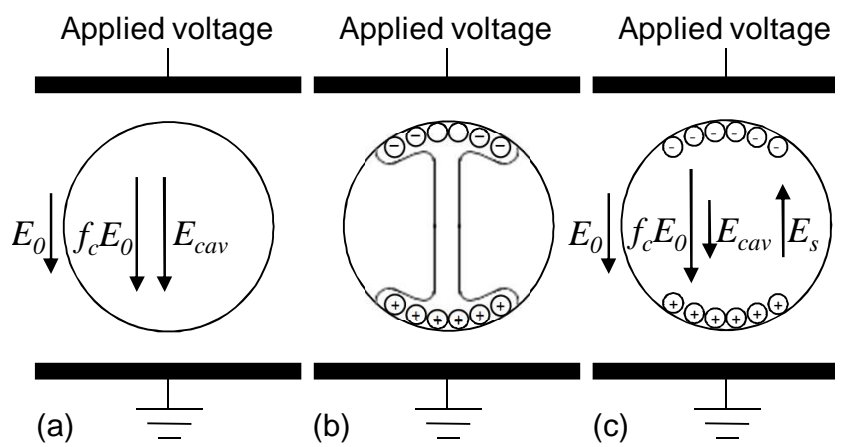

Figure 1. Schematic diagram of a PD event in the cavity: (a) before PD, (b) during PD and (c) after PD.

After a discharge, charges that accumulate on the cavity surface may become trapped in surface states or remain free on the cavity wall. Free charges may move along the cavity wall through surface conduction [13]. The movement of free surface charges that have accumulated along the cavity wall after a PD has completely occurred is assumed to be dependent on the magnitude of electric field in the cavity, $E_{\text {cav }}$ and the field due to the surface charge, $E_{s}$ and their polarities with respect to each other [14]. This will affect the cavity surface charge distribution. Referring to figure 1(c), when the polarity of $E_{c a v}$ is opposite to that of $E_{s}$, free surface charge tends to move towards the symmetry axis of the cavity. However, when the polarity of $E_{\text {cav }}$ is the same as $E_{s}$, surface charge tends to move towards the opposite sign charge along the cavity wall, ultimately resulting in charge recombination. Thus, the amount of free surface charge and the magnitude of field due to surface charge decrease over time.

\section{Experiment design}

The experiment setup that has been used for the PD measurement consists of a high voltage supply, $V$, a coupling capacitor $C_{k}$ (capacitance of $1 \mathrm{nF}$ ), a test sample (equivalent capacitance of $0.01 \mathrm{pF}$ ), a measuring impedance, a PD detector and a USB controller which is connected to a personal computer (PC) (figure 2(a)). Discharge signals from the test sample are detected by the measuring impedance and the PD detector. The measuring impedance can be represented by a simplified $R L C$ circuit, consisting of equivalent resistance, $R(200 \Omega)$, capacitance, $C(1 \mathrm{nF})$ and inductance, $L$ $(0.1 \mathrm{uH})$. The output from the PD detector is sent to the PC through USB controller for user data analysis. 
Figure 2(b) shows the output current of a $50 \mathrm{~Hz}, 7 \mathrm{kV}$ peak amplitude ac sinusoidal appllied voltage across a $500 \mathrm{k} \Omega$ resistor which is connected in series with the high voltage supply without connecting to the test object and the PD detector. For any applied voltage amplitude, the output current has the waveform shape as in figure 2(b) with respect to the applied voltage.

The test sample consists of an artificial spherical cavity located in the middle of a cylindrical epoxy resin with a relative permittivity, $\varepsilon_{r}$ of 4.4 (figure 3 ). First, an air bubble was injected into a small block of an epoxy (thickness, $h_{\text {small }}$ ) before curing to obtain a spherical cavity (radius, $r_{c a v}$ ). The small block was cast into a larger epoxy block (thickness, $h_{\text {mat }}$ ) before curing for 24 hours at room temperature. Then, post curing for 4 hours at $90^{\circ} \mathrm{C}$ was undertaken before allowing the sample to cool down to ambient temperature for 2 hours. The top and bottom surfaces of the test object were placed in contact with a cylindrical electrode (diameter of $25 \mathrm{~mm}$ ) each, where the top electrode was connected to a high voltage supply while the bottom electrode was always grounded. The whole test sample and the electrodes were immersed in mineral oil during the experiment to prevent discharge around the edge of the electrodes. A 50 $\mathrm{Hz}$, ac sinusoidal voltage was applied to the sample with a variable peak amplitude of 14 to $20 \mathrm{kV}$. For all PD measurements, PD data over 500 applied voltage cycles were recorded and simulation were also performed for 500 applied voltage cycles.

It cannot be guaranteed that the shape of the cavity obtained is a perfect spherical. However, it is assumed that the shape of the cavity is close to that of perfect spherical. This assumption is based on the observation of the cavity under a microscope with light, where the largest circumference of the cavity has been found to be a perfect circle. When the cross section of the sample containing the cavity is observed by cutting the sample into two semi cylindrical, the circumference of the cavity has also been found to be a perfect circle. Hence, it is assumed that shape of the cavity obtained is close to a perfect spherical. Moreover, this is the standard way for preparing a spherical cavity in a dielectric material that has been used in previous works $[3,4]$. In this work, the measurement results that have been obtained using all samples that have been prepared have the similar characteristics of the measurement results from previous works.

\section{PD model}

\subsection{FEA model geometry}

The model uses a two-dimensional (2D) axial symmetric, electrostatic module in Finite Element Analysis (FEA) software, which is solved for local electric fields and is used in parallel with MATLAB code. The governing partial differential equation that is solved using FEA method is

$$
\vec{\nabla} \bullet \varepsilon \vec{E}=\rho
$$

where $\varepsilon$ is the permittivity, $E$ is the electric field and $\rho$ is the charge density. 


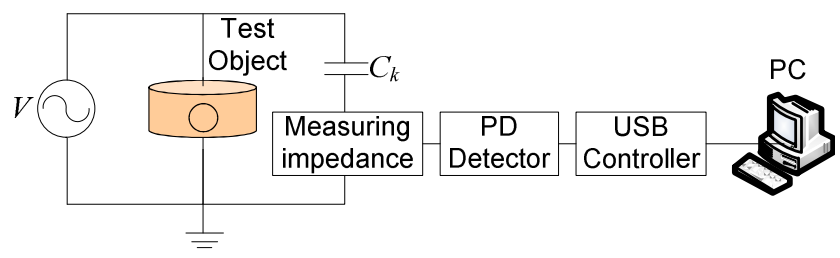

(a)

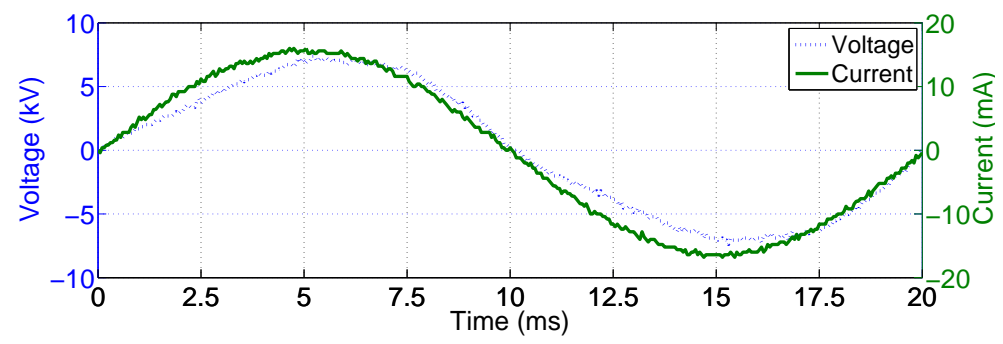

(b)

Figure 2. (a) Schematic diagram of the experiment setup and (b) the output current of the high voltage ac supply.

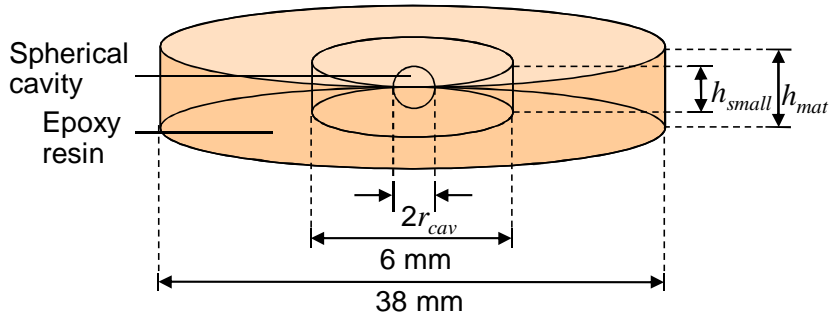

Figure 3. Schematic diagram of the test sample prepared.

The 2D axial symmetric model geometry is detailed in figure 4 . It consists of a homogenous dielectric material (thickness, $h_{m a t}=2 \mathrm{~mm}$, radius $=5 \mathrm{~mm}, \varepsilon_{r}=4.4$ ) and a hemispherical cavity (radius, $r_{c a v}=0.7 \mathrm{~mm}, \varepsilon_{r}=1$ ). A $50 \mathrm{~Hz}$, ac sinusoidal voltage is applied to the upper electrode while the lower electrode is always grounded. The upper and lower cavity surfaces are divided into ten equal regions to model the surface charge distribution along the cavity wall. The surface region of the upper and lower cavity surfaces nearest to the symmetry axis is referred as surface region 1 while the surface region where the upper and lower cavity surfaces meet is referred to as region 10 . The area of each surface region is the same to ensure that the charge density and the amount of charge propagation on each surface region are identical. The cavity surface area, $A$ of each of the 20 surface regions equal to

$$
A=4 \pi r_{c a v}^{2} / 20=\pi r_{c a v}^{2} / 5
$$

\subsection{Partial discharge model}

In this model, PD is assumed to occur from the cavity surface that is on the symmetry axis, passing through the cavity along the symmetry axis and reaching the other side 


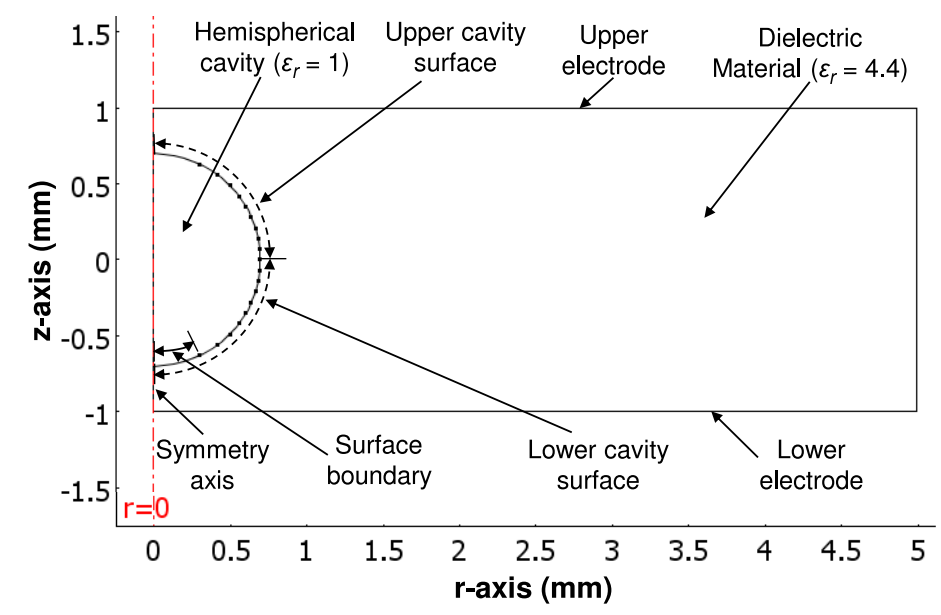

Figure 4. 2D axial symmetric model geometry.

of the cavity surface. For a spherical cavity, the electric field on the cavity surface that is perpendicular to the direction of the applied field is highest. This is due to charge density accumulation on that cavity surface is highest. Thus, the likelihood of a PD occurrence from the cavity surface that is perpendicular to the direction of the applied field (on the symmetry axis) will be very high. Therefore, for the purposes of modelling, PD is only assumed to occur between cavity surfaces that are on the symmetry axis.

For the first PD to occur, the field at the cavity inner surface close to the electrodes at an instantaneous time $t, E_{\text {cav }}\left(t, 0, r_{\text {cav }}\right)$ must exceed the inception field, $E_{\text {inc }}$, where $\left(0, r_{\text {cav }}\right)$ represents the location of the upper cavity inner surface close to the electrode. Prior to the first PD event, the electric field is lowest at the cavity inner surface areas closest to the electrodes. However, for subsequent discharges, the electric field in the cavity at its centre, $E_{\text {cav }}(t, 0,0)$ is extracted from the model geometry to determine whether a PD will occur or not, where $(0,0)$ represents the location of the centre of the cavity. The field in the centre of the cavity is lowest after the first PD has occurred. When the field in the centre of the cavity at an instantaneous time $t, E_{c a v}(t, 0,0)$ exceeds the inception field, $E_{\text {inc }}$, a subsequent discharge may occur. $E_{c a v}$ is the average field along the symmetry axis of the cavity. $E_{i n c}$ is constant (assuming gas pressure in the cavity is constant) and depends on the distance across the cavity symmetry axis, i.e. the cavity diameter.

During a PD event, once the charge streamer has passed through the cavity to the opposite surface, discharge is assumed to propagate on the upper and lower cavity surface. The charge propagation on the upper and lower cavity surfaces is assumed to be symmetrical and identical. Thus, charge distribution on the cavity surface is symmetrical along the $r$ and $z$ axes. The number of surface regions from the symmetry axis of the upper and lower cavity surfaces at which charges propagate is determined through comparison of real charge magnitude, $q_{\text {real }}$ obtained from the FEA model in this work and reports from other researchers [3, 4]. The field change in the cavity due to a discharge, $E_{c a v}$ from the FEA model determines the real charge magnitude, $q_{\text {real }}$ 
which is calculated using $[3,4]$

$$
q_{\text {real }}=\varepsilon_{0} \pi r_{\text {cav }}^{2}\left(1+2 \varepsilon_{r}\right) \Delta E_{\text {cav }}
$$

where $\varepsilon_{0}=8.85 \times 10^{-12} \mathrm{Fm}^{-1}$ and $\Delta E_{\text {cav }}$ is the field change due to a PD.

Initially, before a PD occurs, the charge density on each surface region is equal to zero. During the first discharge, when charges arrive on the cavity surface and start to propagate along the cavity surface, a surface charge density, $\rho_{s}(t, r, z)$ is added at each surface region where charges propagate. If the direction of the electric field in the cavity centre, $E_{\text {cav }}(t, 0,0)$ is from the upper to lower cavity surface, a charge density, $+\rho_{s}(t, r, z)$ is added on the lower cavity surface and $-\rho_{s}(t, r, z)$ is added on the upper cavity surface. The polarity for $\rho_{s}(t, r, z)$ changes when the direction of $E_{c a v}(t, r, z)$ is from the lower to upper cavity surface.

To determine the amount of charge due to a discharge, during the discharge event, a surface charge density, $\rho_{s}\left(t_{n}, r, z\right)$ is added on each surface region where charges propagate using

$$
\rho_{s}\left(t_{n}, r, z\right)=\rho_{s 0}\left[1+\frac{\left|E_{c a v}\left(t_{n}, 0,0\right)\right|-\left|E_{\text {ext }}\right|}{\left|E_{\text {inc }}\right|-\left|E_{\text {ext }}\right|}\right]
$$

where $\rho_{s 0}$ is the initial surface charge density, $E_{\text {inc }}$ and $E_{\text {ext }}$ are the discharge inception and extinction fields in the middle of the cavity and $n$ is the $n$-th time step during the discharge event. At each time step during PD event, the increment of $\rho_{s}\left(t_{n}, r, z\right)$ on the cavity surface causes $E_{c a v}\left(t_{n}, 0,0\right)$ to decrease. Thus, the value of $\rho_{s}$ on the cavity surface at each time step is smaller when $E_{\text {cav }}\left(t_{n}, 0,0\right)$ decreases, while $\left|E_{\text {inc }}\right|-\left|E_{\text {ext }}\right|$ remains constant. $\rho_{s}\left(t_{n}, r, z\right)$ is added until $E_{\text {cav }}\left(t_{n}, 0,0\right)$ becomes less than $E_{\text {ext }}$, when discharge stops. Therefore, setting $\rho_{s}\left(t_{n}, r, z\right)$ as dependent on $E_{\text {cav }}\left(t_{n}, 0,0\right)$ and $E_{\text {ext }}$ in (4) allows a PD to stops at field level very close to $E_{\text {ext }}$. When the next PD occurs, $\rho_{s}\left(t_{n}, r, z\right)$ is added to the charge density that is still remaining on the cavity surface region from the previous discharge.

The PD real charge magnitude, $q_{\text {real }}$ is calculated by summation of charge density that has been added at the cavity surface regions where charges have propagated during the discharge are multiplied by the cavity surface area, $A$ which is obtained from (2), i.e.

$$
q_{\text {real }}=A \sum_{k=1}^{m}\left[\sum_{n=1} \rho_{s k}\left(t_{n}, r, z\right)\right]
$$

where $k$ is the $k$-th surface region, $m$ is the total number of surface regions where charges have propagated during the discharge event and $\rho_{s k}\left(t_{n}, r, z\right)$ is surface charge density on $k$-th region at $n$-th time step.

The PD apparent charge magnitude, $q_{a p p}$ is calculated by integration of the difference between surface charge density on the ground electrode before, $\rho_{s e}^{\prime}(r, z)$ and after, $\rho_{s e}^{\prime \prime}(r, z)$ a discharge over the ground electrode surface area, where

$$
q_{a p p}=\int\left(\rho_{s e}^{\prime \prime}(r, z)-\rho_{s e}^{\prime}(r, z)\right) d S
$$


This represents the magnitude of the PD event measured at the electrodes of the test samples and will be less than the PD real charge of the void.

\subsection{Charge movement through surface conduction}

After a PD, some charges become trapped in surface states and some remain free on the cavity surface. Charges that remain on the surface may move freely along the cavity surface, depending on the direction and magnitude of the electric field in the cavity. In order to determine the amount of charge that remains free on the $k$-th cavity region immediately after a discharge, $q_{s f k}$, the electric displacement field on each surface region where charges have propagated during a discharge is integrated over the upper cavity surface area covered by a surface area, $A$,

$$
q_{s f k}\left(t_{P D}, r, z\right)=\int_{A}\left(D_{z k(u p)}-D_{z k(\text { down })}\right) d S
$$

where $t_{P D}$ is the time of a PD occurrence and $D_{z k(u p)}$ and $D_{z k(\text { down })}$ are the $z$-component of the displacement field on the top and bottom of each upper cavity surface region. Thus, the free charge density, $\rho_{s f k}\left(t_{P D}, r, z\right)$ on each surface region where charges have propagated is calculated using

$$
\rho_{s f k}\left(t_{P D}, r, z\right)=q_{s f k}\left(t_{P D}, r, z\right) / A
$$

When charge propagation during discharge is only within certain regions of the cavity surface, the charge distribution becomes non-uniform because of the variation in surface charge density. Thus, the movement of free charge on the cavity surface is set as dependent on the polarity of the electric field, $E_{\text {cavk }}(t, r, 0)$ and the polarity of surface charge, $E_{s k}(t, r, 0)$ in each cavity surface region on $r$ axis $(z=0)$, where $k$ is the surface region number.

When the polarity of $E_{\text {cavk }}(t, r, 0)$ is the same as $E_{s k}(t, r, 0)$, free charges on surface region $k$ of the upper and lower cavity surfaces are assumed to move away from the cavity symmetry axis through charge conduction along the cavity wall. It is assumed that the movement of charge on the upper cavity surface is mirrored by movement on the lower cavity surface. To model this, the change in the charge density, $\Delta \rho_{s k}\left(t_{n}, r, z\right)$ on each surface region at $n$-th time step is defined as [13]

$$
\Delta \rho_{s k}\left(t_{n}, r, z\right)=\frac{1}{A} \sigma_{s} \int_{c}\left[E_{s t k}\left(t_{n}, r, z\right) d S\right] \Delta t
$$

where $\sigma_{s}$ is the cavity surface conductivity, $c$ is a curve that bounds a cavity surface area $A, E_{s t k}\left(t_{n}, r, z\right)$ is the tangential field along the $k$-th cavity surface region at $n$-th time step and $\Delta t$ is the time step interval when a PD is not occurring. Only if $\Delta \rho_{s k}\left(t_{n}, r, z\right)$ is less than $\rho_{s f k}\left(t_{P D}, r, z\right)$, the calculated value from (9) is considered. Else, $\Delta \rho_{s k}\left(t_{n}, r, z\right)$ is set equal to $\rho_{s f k}\left(t_{P D}, r, z\right) . \sigma_{s}$ is assumed to be the same for the whole cavity surface. Its value may vary depending on the applied stress conditions. Thus, the surface charge density left on each cavity surface region after the charge movement can be defined as

$$
\rho_{s k}\left(t_{n}, r, z\right)=\rho_{s k}\left(t_{n-1}, r, z\right)-\Delta \rho_{s k}\left(t_{n}, r, z\right)
$$


where $k=1,2, \ldots, 10$.

The charge that has moved from a surface region will move into the adjacent surface region that is further from the symmetry axis. Therefore, the adjacent surface region will become

$$
\rho_{s(k+1)}\left(t_{n}, r, z\right)=\rho_{s(k+1)}\left(t_{n-1}, r, z\right)+\Delta \rho_{s k}\left(t_{n}, r, z\right)
$$

where $k=1,2, \ldots, 9$.

At the point where the upper and lower cavity surfaces meets, charge recombination occurs, resulting in a decrease of the total amount of surface charge on the cavity wall. However, when the polarity of $E_{c a v k}(t, r, z)$ is opposite to that of $E_{s k}(t, r, z)$, surface charge tends to move towards the symmetry axis of the cavity and is assumed to concentrate on region1 of the cavity surface. In this case, free charge on a surface region will move to the adjacent region that is nearer to the symmetry axis. The same equations as (9) and (10) are used but (11) is modified slightly, where

$$
\rho_{s(k-1)}\left(t_{n}, r, z\right)=\rho_{s(k-1)}\left(t_{n-1}, r, z\right)+\Delta \rho_{s k}\left(t_{n}, r, z\right)
$$

where $k=2,3, \ldots, 10$.

Equations (9) to (12) are calculated only when charge density on a surface region where charge has propagated is more than the trapped charge density on that surface region immediately after a $\mathrm{PD}$ occurs. It is due to the trapped charges that cannot move freely along the cavity surface. The trapped charge density on those surface regions, $\rho_{s t k}\left(t_{P D}, r, z\right)$ at $t_{P D}$ is calculated using

$$
\rho_{s t k}\left(t_{P D}, r, z\right)=\rho_{s k}\left(t_{P D}, r, z\right)-\rho_{s f k}\left(t_{P D}, r, z\right)
$$

where $\rho_{s k}\left(t_{P D}, r, z\right)$ is the total surface charge density and $\rho_{s f k}\left(t_{P D}, r, z\right)$ is the free surface charge density, calculated using (8).

Therefore, when free charges move along the cavity wall through surface conduction, the surface charge distribution becomes non-uniform. This influences the electric field distribution in the cavity and the material. The electric field in the cavity centre, $E_{c a v}(t, 0,0)$ and the net field due to the surface charge in the cavity centre, $E_{s}(t, 0,0)$ are affected and this in turn influences the likelihood of the next PD event occurring.

Free surface charge remaining on the cavity surface may also decay through volume conduction, where the decay rate depends on the material conductivity [13]. However, in this study, this mechanism has been neglected because the charge decay rate is very slow compared to the period of the applied voltage. Thus, this mechanism does not have significant effect on surface charge distribution over each half cycle of the applied voltage.

\subsection{Modelling of PD occurrence}

When the field in the cavity center, $E_{\text {cav }}(t, 0,0)$ has exceeded the inception field, $E_{\text {inc }}$, an initial free electron is required for a PD to occur. The amount of initial free electron 
available due to surface emission is assumed to be dependent only on the detrapping of charge from the cavity surface that has been trapped along the cavity surface region due to previous PD event. It is the main source of electron generation rate (EGR) through surface emission $[3,4]$. Since PD is only assumed to occur along the symmetry axis of the cavity, EGR becomes a function of time only. The amount of charge detrapping for the next $\mathrm{PD}$ event is assumed to be dependent on the field in the cavity centre of previous PD occurrence, $E_{c a v}\left(t_{P D}, 0,0\right)$, where $t_{P D}$ is the time of previous PD occurrence. If $N_{e s 0}$ is the number of free electrons generated in the cavity per unit time at $E_{\text {inc }}$, the number of free electrons generated in the cavity through surface emission per unit time due to the previous PD event, $N_{P D}$ for the next discharge is likely to occur can be defined as

$$
N_{P D}=N_{e s 0}\left|E_{c a v}\left(t_{P D}, 0,0\right) / E_{\text {inc }}\right|
$$

$N_{P D}$ is assumed to be linearly dependent on the $E_{c a v} / E_{\text {inc }}$, where $N_{P D}$ has been assumed to be dependent on the amount of charge that has accumulated on the cavity surface due to the previous PD event. The amount of charge accumulated on the cavity surface due to the previous PD event is equal to the real charge magnitude, which is linearly proportional to the field change, $\Delta E$ in the cavity due to a PD event (where $\left.\Delta E=E_{\text {cav }}-E_{\text {ext }}\right)$. If $E_{\text {ext }}$ is assumed to be constant throughout the simulation, the real charge magnitude is linearly proportional to $E_{c a v}$. Thus, $N_{P D}$ is linearly proportional to $E_{\text {cav }}$. To derive a relationship between $N_{P D}$ and $E_{c a v}, N_{P D}$ is assumed to be equal to $N_{e s 0}$ when $E_{c a v}$ is equal to the inception field, $E_{i n c}$. This is derived based on the assumption that the electron generation rate due to surface emission is lowest at $E_{\text {inc }}$. If the previous PD occurs at a higher field level (higher $E_{\text {cav }}$ ) than $E_{\text {inc }}$, the electron generation rate due to surface emission for the next PD to occur is increased. Therefore, $N_{P D}$ is assumed to be linearly proportional to $E_{\text {cav }} / E_{\text {inc }}$.

In order to consider charge decay of the amount of trapped charge near the cavity surface from the previous PD event, which is likely to be detrapped at the time when the next PD is likely to occur, it is assumed that the charge decay is exponential [3]- [5]. If EGR due to surface emission is assumed to increase exponentially with electric field, the complete equation for EGR due to surface emission, $N_{e s}(t)$ can be defined as

$$
N_{e s}(t)=N_{P D} \exp \left[-\left(t-t_{P D}\right) / \tau_{d e c}\right] \exp \left|E_{c a v}(t, 0,0) / E_{\text {inc }}\right|
$$

where $\tau_{d e c}$ is the effective charge decay time constant and $\left(t-t_{P D}\right)$ is the elapsed time since the previous PD. $\left|E_{\text {cav }}(t, 0,0) / E_{\text {inc }}\right|$ represents simplified field dependent term for $N_{e s}(t)$. A higher field in the cavity, $E_{c a v}(t, 0,0)$ increases the EGR due to surface emission. The value for $N_{e s 0}$ is divided into two, a higher value, $N_{e s 0 H}$ and a lower value, $N_{e s 0 L}$. When the next PD may occur and the polarity of the field in the cavity, $E_{c a v}(t, 0,0)$ is opposite to that during the previous PD, $E_{c a v}\left(t_{P D}, 0,0\right)$, the cavity surface work function is assumed to be higher because electrons will be detrapped from a negative charge cavity surface $[3,4]$. Hence, the chances of electron detrapping from the cavity surface are lower, requiring $N_{e s 0 L}$ to be used. However, the chances of electron detrapping are higher when the polarity of $E_{c a v}(t, 0,0)$ and $E_{c a v}\left(t_{P D}, 0,0\right)$ are identical 
because electrons will be detrapped from a positive cavity surface charge and the work function will be reduced. Thus, $N_{e s 0 H}$ is used.

Initial free electrons may also be generated through volume ionization, which is independent of the cavity surface charge distribution [3]-[5]. The EGR due to volume ionization, $N_{e v}$ is defined as the number of free electrons generated in the cavity per unit time through volume ionization. $N_{e v}$ is a constant to simplify the model, which depends on the applied stress and cavity size. Thus, the total EGR at an instantaneous time $t, N_{e t}(t)$ can be defined as a summation of EGR due to surface emission, $N_{e s}(t)$ from (15) and EGR due to volume ionization, $N_{e v}$,

$$
N_{e t}(t)=N_{e s}(t)+N_{e v}
$$

Note that $N_{e t}(t)$ only depends on $N_{e v}$ for a cavity that has yet to experience any PD, as there is an absence of trapped charge near the cavity surface. After the first PD occurs, $N_{e t}(t)$ for the next PD depends on both $N_{e s}(t)$ and $N_{e v}$.

The availability of an initial free electron is modeled using a likelihood of a PD occurrence, $L(t)$ which is assumed as dependent on $N_{e t}(t)$ and the time stepping interval, $\Delta t_{0}$ during no PD event. $L(t)$ is compared with a random number, $R$ (between 0 to 1 ). A PD will occur only if $L(t)>R$. $L(t)$ is defined as [4]

$$
L(t)=N_{e t}(t) \Delta t_{0}
$$

Table 1 shows all simulation parameters that has been used for the whole simulation while table 2 shows simulation parameters that depends on the stress and cavity conditions. For the time step during no $\mathrm{PD}, \Delta t_{0}$, the field in the cavity centre, $E_{\text {cav }}(t, 0,0)$ will change too fast with one time step if it is set too long, resulting in less precision of the phase of $\mathrm{PD}$ occurrence. However, when $\Delta t_{0}$ is set too short, the simulation time will be increased greatly. Therefore, a suitable value has been chosen to keep the simulation time reasonable but still ensuring that the precision of phase of PD occurrence is good. The time interval during PD event, $\Delta t_{1}$ has no influence on the simulation results at all. Therefore, it is set to a very small value so that the applied field magnitude does not change significantly during PD occurrence. The material permittivity, $\varepsilon_{\text {rmat }}$ used in the simulation is obtained from laboratory measurements. The cavity permittivity, $\varepsilon_{\text {rcav }}$ is set equal to 1 because it is assumed that the content in the cavity is air. The effective charge decay time constant, $\tau_{d e c}$ was $2 \mathrm{~ms}$ as reported in [4], $\tau_{\text {dec }}$ only depends on material temperature.

Referring to table 2, parameters relating to electron generation rate, i.e. $N_{e s 0 H}$, $N_{e s 0 L}$ and $N_{e v}$ do not directly represent physical properties of the dielectric material. Electron generation rate is dependent on a large range of factors and to model this in terms of physical propeeties would require significant computation. Instead, the electron generation rate is defined through comparison with experimental results. This model parameter is dependent on both the applied stress and cavity condition.

The values of $N_{e s 0 H}, N_{e s 0 L}$ and $N_{e v}$ are dependent on each other. Unsuitable choice of values will yield significant error between measurement and simulation results. For 
Table 1. Definition and symbol of parameters used for the whole simulation.

\begin{tabular}{llll}
\hline Definition & Symbol & Value & Unit \\
\hline Applied frequency & $f$ & 50 & $\mathrm{~Hz}$ \\
Time step during no PD & $\Delta t_{0}$ & $1 / 360 f$ & $\mathrm{~s}$ \\
Time step during PD & $\Delta t_{1}$ & 1 & $\mathrm{~ns}$ \\
Material relative permittivity & $\varepsilon_{\text {rmat }}$ & 4.4 & \\
Cavity relative permittivity & $\varepsilon_{\text {rcav }}$ & 1 & \\
Effective charge decay time constant & $\tau_{\text {dec }}$ & 2 & $\mathrm{~ms}$ \\
\hline
\end{tabular}

Table 2. Stress and cavity condition-dependent parameters in the simulation.

\begin{tabular}{lll}
\hline Definition & Symbol & Unit \\
\hline Cavity radius & $r_{c a v}$ & $\mathrm{~mm}$ \\
Material thickness & $h_{m a t}$ & $\mathrm{~mm}$ \\
Applied field & $E_{0}$ & $\mathrm{kVmm}^{-1}$ \\
Initial surface charge density & $\rho_{s 0}$ & $\mathrm{Cm}^{-2}$ \\
Cavity surface conductivity & $\sigma_{s}$ & $\mathrm{Sm}^{-1}$ \\
Inception field & $E_{\text {inc }}$ & $\mathrm{kVmm}^{-1}$ \\
Extinction field & $E_{e x t}$ & $\mathrm{kVmm}^{-1}$ \\
Higher initial EGR due to surface emission & $N_{e s 0 H}$ & $\mathrm{~s}^{-1}$ \\
Lower initial EGR due to surface emission & $N_{e s 0 L}$ & $\mathrm{~s}^{-1}$ \\
Electron generation rate due to volume ionization & $N_{e v}$ & $\mathrm{~s}^{-1}$ \\
\hline
\end{tabular}

example, if the electron generation rate is set too high, the PD patterns of the simulation will not be comparable to the measured patterns and the simulated number of PDs per cycle will be too large. However, if the electron generation rate is set too low, it will result in no PD occurring for many voltage cycles, consequently resulting in a very low simulated number of PDs per cycle. Therefore, sensitivity analysis was performed to select the optimum values of $N_{e s 0 H}, N_{e s 0 L}$ and $N_{e v}$, using an optimization method once the other parameter values in table 2 had been determined. For each possible combination of $N_{e s 0 H}, N_{e s 0 L}$ and $N_{e v}$, the total mean square error (MSE) between simulation and measurement in terms of the number of PDs per cycle against the phase of PD occurrence distribution, $H_{n}$, and the total charge per cycle against the phase of PD occurrence distribution, $H_{q s}$, were calculated. The least MSE from all combinations of $N_{e s 0 H}, N_{e s 0 L}$ and $N_{e v}$ was identified and these values were assumed for all simulations performed.The acceptance criteria used to validate the choice of parameter value are that the average number of PDs per cycle between measurement and simulation must be \pm 0.1 and there must be less than $10 \%$ difference for the total charge per cycle or maximum PD charge magnitudes. 


\section{Results and Discussion}

Comparison of simulation and measurement results as a function of applied voltage amplitudes and cavity sizes has been undertaken to validate the model. Field distribution plots from the FEA model that has been developed are presented in the section. Measurement results as a function of applied field and cavity radius are also explained in this section.

\subsection{Electric field distribution}

For the geometry shown in figure 4 , it has been found that only regions 1 to 5 of the upper and lower cavity surfaces has increased surface charge density during a discharge event which matches the $q_{\text {real }}$ obtained from the FEA model with the $q_{\text {real }}$ calculated from (3) for the same $\Delta E_{c a v}$. Therefore, charges only propagate along the surface regions 1 to 5 during a discharge event. This may indicate that the field of charge propagation along the cavity wall may not be strong enough for charge to continue propagating into surface region 6 , which effectively terminating further charge propagation. In addition, charge deposition in regions 6 to 10 of the cavity surface does not contribute significantly to PD apparent charge magnitude. Hence, it is possible that charge will only propagate on regions 1 to 5 of the cavity surface during PD event.

The simulation of electric field distribution and electric equipotential lines before, immediately after and $0.02 \mathrm{~s}$ after the first PD occurrence are shown in figure 5 while table 3 shows the simulation parameters that has been used to obtain these figures. The values for $E_{i n c}, E_{\text {ext }}, \rho_{s 0}$ and $\sigma_{s}$ used for these figures are obtained from the simulation detailed in the next section. In figure 5(a), the electric field before the first PD is higher in the cavity than in the surrounding dielectric material because the relative permittivity of the cavity is lower than that of the solid dielectric. The electric field distribution in the whole cavity is nearly uniform before the first PD occurs, where the electric field is slightly lower on the cavity surface areas closest to the electrodes because the applied field is almost perpendicular to the cavity surface [15].

When the first PD occurs after the field in the cavity is greater than the inception

field, $E_{i n c}$, surface charge density on surface regions 1 to 5 of the upper and lower cavity surfaces increases, causing the electric field in the cavity to decrease. However, the electric field on the cavity surface nearest to the electrode is significantly increased due to charge accumulation on the cavity surface, as shown in figure 5(b). Since no charge accumulates on the cavity wall in regions 6 to 10, the electric field along that cavity wall is less affected by the discharge. Electric field distribution in the cavity after a PD will be non-uniform and affect the occurrence of the next PD event.

When the accumulated surface charge moves away from the symmetry axis through surface conduction along the cavity wall, the electric field in the cavity is modified, as shown in figure 5. There is charge recombination on the cavity surface, resulting in a reduction of the total surface charge. The electric field on the cavity surface nearest to the electrode is reduced and the field in the whole cavity becomes more uniform. 

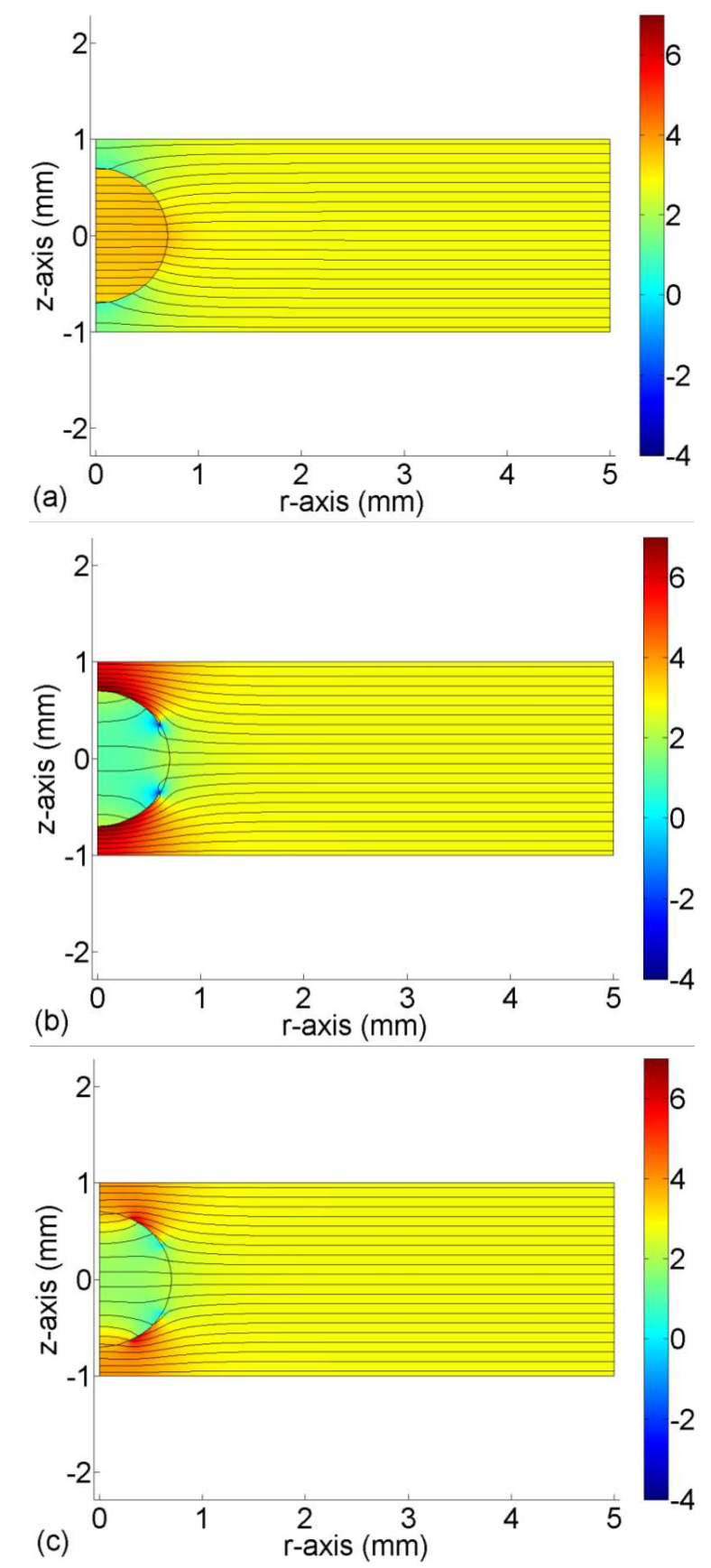

Figure 5. Electric field distribution (surface plot $\left[\mathrm{kVmm}^{-1}\right]$ ) and electric equipotential lines (contour plot) from the FEA model: (a) before a PD event, (b) after a PD event and (c) $0.02 \mathrm{~s}$ after a PD event.

From the simulation, if the first discharge occurs after $5 \mathrm{~ms}$ and different surface conductivities, $\sigma_{s}$ are used, then the decay rate of electric field in the cavity centre due to surface charge, $E_{s}(t, 0,0)$ can be modelled (figure 6 ). When charge moves along the cavity wall, the electric field distribution in the cavity is altered. As $\sigma_{s}$ increases, charge moves faster along the cavity wall, resulting in faster decay of the amount of surface charge through charge recombination and faster reduction of $E_{s}(t, 0,0)$ with time. 
Table 3. Simulation parameters used in the model for figure 5.

\begin{tabular}{llll}
\hline Definition & Symbol & Value & Unit \\
\hline Cavity radius & $r_{\text {cav }}$ & 0.7 & $\mathrm{~mm}$ \\
Material thickness & $h_{\text {mat }}$ & 2 & $\mathrm{~mm}$ \\
Applied field & $E_{0}$ & 9 & $\mathrm{kVmm}^{-1}$ \\
Initial surface charge density & $\rho_{s 0}$ & $1 \times 10^{-4}$ & $\mathrm{Cm}^{-2}$ \\
Cavity surface conductivity & $\sigma_{s}$ & $5.6 \times 10^{-9}$ & $\mathrm{Sm}^{-1}$ \\
Inception field & $E_{\text {inc }}$ & 3.41 & $\mathrm{kVmm}^{-1}$ \\
Extinction field & $E_{\text {ext }}$ & 1.1 & $\mathrm{kVmm}^{-1}$ \\
\hline
\end{tabular}

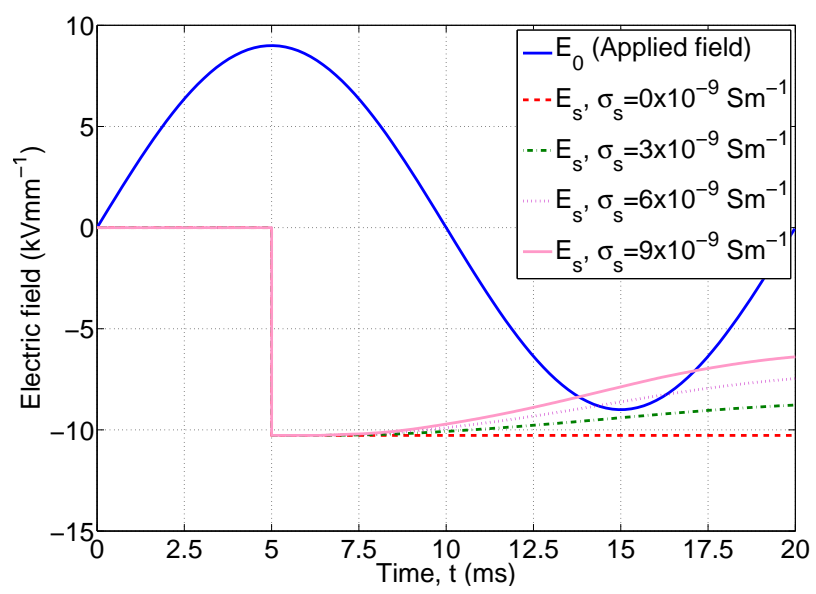

Figure 6. Simulation of electric field due to surface charge, $E_{s}$ as a function of time using different surface conductivity (All simulation parameters are the same as in table $3)$.

The simulation of the electric field and PD charge magnitude over the first two cycles of applied voltage are shown in figure 7 [17] and the simulation parameters used shown in table 4 . The values for $E_{i n c}, E_{e x t}, \rho_{s 0}, \sigma_{s}, \tau_{d e c}, N_{e s 0 H}, N_{e s 0 L}$ and $N_{e v}$ are obtained from simulation results that are described in the next section. In order to validate the simulation result, the measurement of $\mathrm{PD}$ apparent charge magnitude with time is shown in Figure 7(c). Initially, before PD occurred, the field in the cavity centre, $E_{\text {cav }}(t, 0,0)$ is equal to the enhanced applied field in the cavity, $f_{c} E_{0}$. After the first PD occurs at $1 \mathrm{~ms}, E_{\text {cav }}(t, 0,0)$ decreases due to the charge accumulated in regions 1 to 5 of the cavity surface produces a field acting in the opposite field direction. This is defined as the field due to surface charge, $E_{s}(t, 0,0)$. The decrease in $E_{c a v}(t, 0,0)$ depends on the amount of charge due to a PD and charge distribution on the cavity surface. When the next PD occurs at 2.1 and $3.3 \mathrm{~ms}, E_{s}(t, 0,0)$ becomes higher due to more charge accumulated on the cavity surface.

When the polarity of $E_{\text {cav }}(t, 0,0)$ is same as that of $E_{s}(t, 0,0)$ (e.g. at 7.2 to $13.3 \mathrm{~ms})$, the magnitude of $E_{c a v}(t, 0,0)$ is enhanced by $E_{s}(t, 0,0)$. Thus, when a PD 


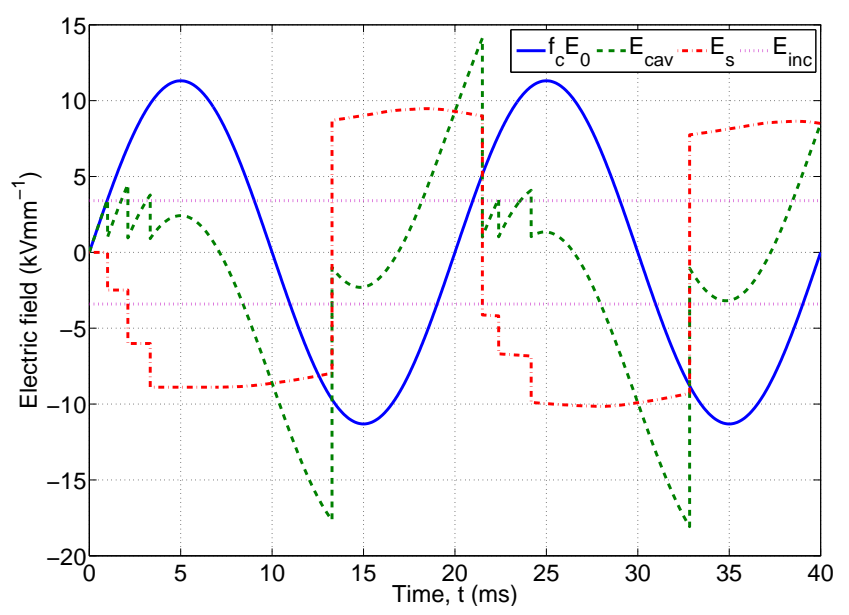

(a)

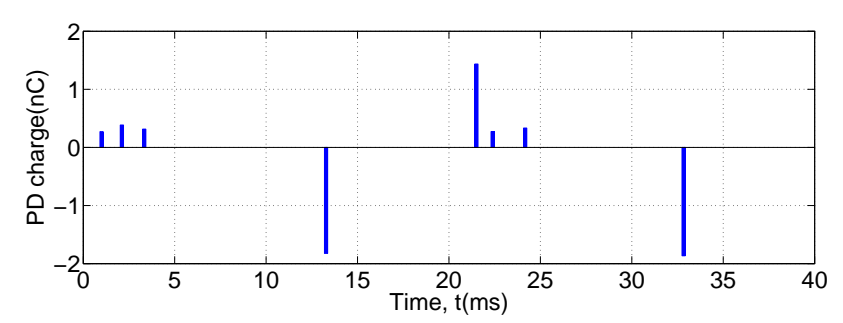

(b)

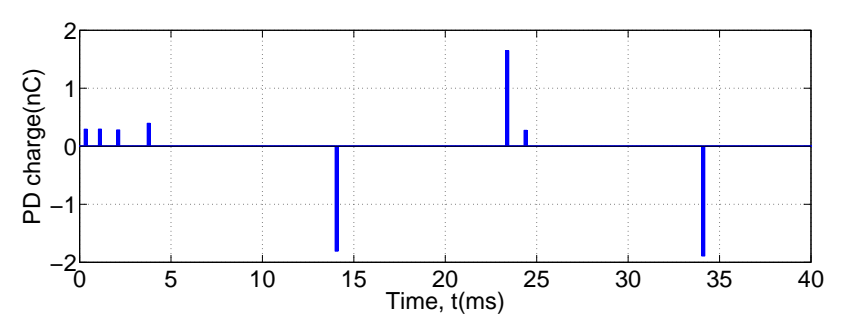

(c)

Figure 7. (a) Simulation of electric fields in the cavity and (b) PD apparent charge magnitude with time; (c) measurement of PD apparent charge magnitude with time.

occurs it has a large charge magnitude due to a larger field existing across the cavity. During this time interval, surface charge moves along the cavity wall through surface conduction, away from the cavity symmetry axis. $E_{s}$ decreases because surface charge is redistributed along the cavity wall. The charge movement towards each other causes charge recombination and a reduction in the total amount of surface charge.

Referring to figure $7(\mathrm{a})$, when the polarity of $E_{c a v}(t, 0,0)$ is opposite to that of $E_{s}(t, 0,0)$ (13.3 to $\left.17 \mathrm{~ms}\right), E_{s}(t, 0,0)$ can be seen to increase. During this time interval, the charge movement along the cavity wall towards the cavity symmetry axis results in an increase of the charge density on the cavity surface near the symmetry axis and $E_{s}(t, 0,0)$ becomes higher. The change in the magnitude of $E_{s}(t, 0,0)$ will affect the PD pattern, especially the curves of the $\phi-q$ - $n$ plot caused by high magnitude of PD events. 
Table 4. Simulation parameters used in the model for figure 7.

\begin{tabular}{llll}
\hline Definition & Symbol & Value & Unit \\
\hline Cavity radius & $r_{\text {cav }}$ & 0.7 & $\mathrm{~mm}$ \\
Material thickness & $h_{\text {mat }}$ & 2 & $\mathrm{~mm}$ \\
Applied field & $E_{0}$ & 9 & $\mathrm{kVmm}^{-1}$ \\
Initial surface charge density & $\rho_{s 0}$ & $1 \times 10^{-4}$ & $\mathrm{Cm}^{-2}$ \\
Cavity surface conductivity & $\sigma_{s}$ & $5.6 \times 10^{-9}$ & $\mathrm{Sm}^{-1}$ \\
Inception field & $E_{\text {inc }}$ & 3.41 & $\mathrm{kVmm}^{-1}$ \\
Extinction field & $E_{e x t}$ & 1.1 & $\mathrm{kVmm}^{-1}$ \\
Initial EGR due to surface emission & $N_{e s 0}$ & $3000\left(N_{e s 0 H}\right), 150\left(N_{e s 0 L}\right)$ & $\mathrm{s}^{-1}$ \\
Electron generation rate due to & $N_{e v}$ & 85 & $\mathrm{~s}^{-1}$ \\
volume ionization & & & \\
\hline
\end{tabular}

When there is no change in polarity of $E_{\text {cav }}(t, 0,0)$ between consecutive discharges, a higher value of $N_{e s 0}$ in (14) is used. Thus, PD occurs immediately after the inception field, $E_{\text {inc }}$ is exceeded (figure 7). However, a lower value of $N_{e s 0}$ is used in (14) when the polarity of $E_{\text {cav }}(t, 0,0)$ changes between consecutive discharges. In this case, PD does not occur immediately after $E_{\text {inc }}$ has been exceeded. These values yield in a simulation result of $\mathrm{PD}$ apparent charge magnitude which agrees with the measurement result (Figure $7(\mathrm{~b}-\mathrm{c}))$.

\subsection{PD behaviour as a function of applied field}

Figure 8 shows the $\phi-q-n$ of measurement results for different applied field and table 5 summarised this PD measurement data. From the PD data of the measurement results, when the applied field is increased, the number of PDs per cycle, the total charge magnitude per cycle and the maximum discharge magnitude increase but the mean charge magnitude decreases. There is a symmetrical behaviour between partial discharges in the negative and positive halves of the ac cycle. This is because the cavity is located near to the middle of the material, thus the electric feld distributions on the upper and lower cavity surface are almost the same. When the applied field is increased, the electric field in the cavity is enhanced and the electron generation rate is increased, which corresponds to a larger number of initial free electrons in the cavity. Therefore, the time delay to obtain an initial free electron to generate PD events decreases, resulting in more PDs occurring earlier in the phase of the applied field. Consequently, there are more PDs per cycle as the applied field is increased [16]. The time delay of getting an initial free electron is defined as the statistical time lag.

The total charge per cycle also increases with increasing applied field because of the higher $\mathrm{PD}$ repetition rate. However, the mean charge magnitude decreases as the applied field is increased because the increase in the number of PDs per cycle is greater 
Table 5. PD data from measurement as a function of applied field.

\begin{tabular}{lllll}
\hline Applied field $\left(\mathrm{kVmm}^{-1}\right)$ & 7 & 8 & 9 & 10 \\
\hline Total PDs per cycle & 2.5 & 4.2 & 5.7 & 7.3 \\
Total charge per cycle $(\mathrm{pC})$ & 1698 & 2591 & 3186 & 3940 \\
Mean charge $(\mathrm{pC})$ & 674 & 616 & 562 & 536 \\
Maximum charge, $q_{\max }(\mathrm{pC})$ & 1625 & 1856 & 2056 & 2250 \\
Minimum charge, $q_{\min }(\mathrm{pC})$ & 263 & 263 & 263 & 263 \\
\hline
\end{tabular}

than the increase in total charge per cycle. The reduction of statistical time lag as the applied field is increased causes more PDs per cycle but they have lower charge magnitudes. The maximum charge magnitude increases with increasing applied field because the higher applied field ensures a larger field reduction in the cavity when a PD occurs.

Referring to the $\phi-q-n$ of the measurement results in figure 8 , for each applied field, there are more PD events occurring near the minimum charge magnitude than at higher charge magnitude. It can be seen that there is a 'rabbit-ear' like curve when looking from the charge magnitude-phase axes, where PDs occur at higher charge magnitudes. $\mathrm{PD}$ events occurring near the minimum charge magnitude are obtained when the field in the cavity does not change polarity between consecutive discharges, as has been shown in figure 7. Due to a smaller time interval between consecutive discharges, the amount of charge decay is less, resulting in a higher electron generation rate when the next PD is likely to occur. Thus, the next PD occurs almost immediately after the inception field has been exceeded, resulting in a lower PD charge magnitude. However, PDs occur with larger charge magnitudes when there is polarity change of the field in the cavity between consecutive discharges. This is due a lower electron generation rate, ensuring that PDs do not occur immediately after the inception field has been exceeded, resulting in a higher PD charge magnitude when a PD occurs.

When the applied field is increased, there are more PD events occurring near the minimum and higher charge magnitudes. The 'rabbit-ear' like curve, by looking on the charge magnitude-phase axes becomes wider with increasing applied field. Due to higher electron generation rate at higher applied field, more PD events per cycle are obtained.

Comparison of PD data between measurement and simulation results as a function of the applied field is shown in table 5 and table 6 while figure 8 shows corresponding simulation $\phi-q-n$ plots. Table 7 shows the simulation parameters that has been used in the model. Measurement and simulation are in reasonable agreement for each applied field. When the applied field is increased, all simulation parameters are kept constant, except the surface conductivity, $\sigma_{s}$ and electron generation rate due to volume ionization, $N_{e v}$, which increase with the applied field.

The simulation value for the inception field, $E_{i n c}$ is chosen based on assessment of measurement data and is assumed independent of the applied field. The extinction 


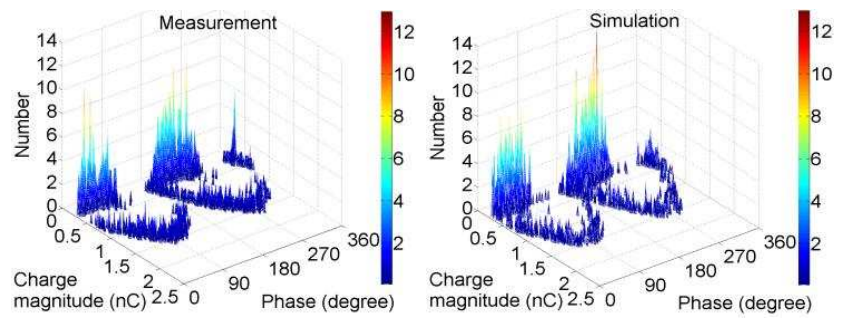

(a)

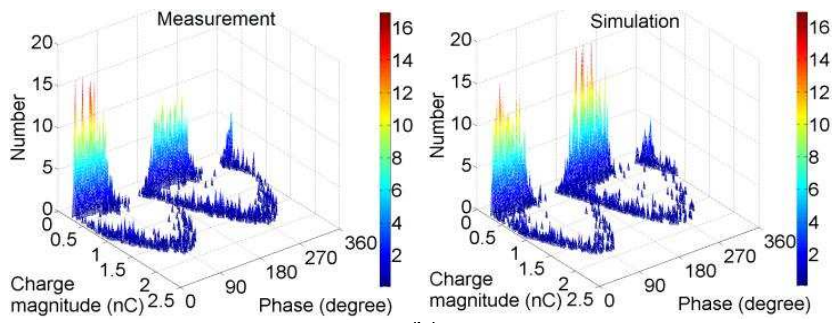

(b)

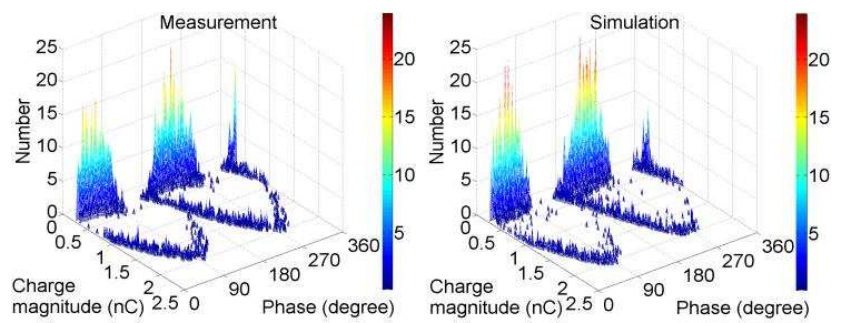

(c)

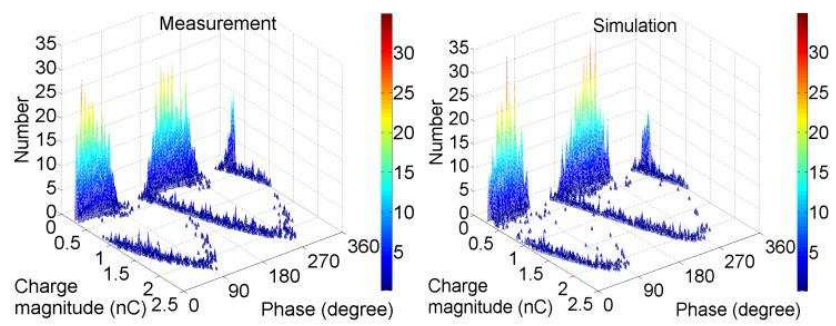

(d)

Figure 8. $\phi-q-n$ plots of measurement and simulation results as a function of applied field magnitude: (a) $7 \mathrm{kVmm}^{-1}$, (b) $8 \mathrm{kVmm}^{-1}$, (c) $9 \mathrm{kVmm}^{-1}$ and (d) $10 \mathrm{kVmm}^{-1}$.

Table 6. PD data from simulation as a function of applied field.

\begin{tabular}{lllll}
\hline Applied field $\left(\mathrm{kVmm}^{-1}\right)$ & 7 & 8 & 9 & 10 \\
\hline Total PDs per cycle & 2.5 & 4.2 & 5.7 & 7.3 \\
Total charge per cycle $(\mathrm{pC})$ & 1372 & 2236 & 3027 & 3832 \\
Mean charge $(\mathrm{pC})$ & 550 & 532 & 533 & 526 \\
Maximum charge, $q_{\max }(\mathrm{pC})$ & 1625 & 1864 & 2064 & 2256 \\
Minimum charge, $q_{\min }(\mathrm{pC})$ & 263 & 263 & 263 & 263 \\
\hline
\end{tabular}

field, $E_{\text {ext }}$ is derived from the measured minimum charge magnitude, $q_{\text {min }}$ where the field drop in the cavity due to a $\mathrm{PD}\left(\Delta E=E_{\text {inc }}-E_{\text {ext }}\right)$ yields $q_{\text {min }}$ in the simulation. $E_{\text {ext }}$ is assumed to be applied field independent since the measured $q_{\min }$ does not change 
Table 7. Simulation parameters used in the model for figure 8.

\begin{tabular}{|c|c|c|c|}
\hline Definition & Symbol & Value & Unit \\
\hline Cavity radius & $r_{c a v}$ & 0.7 & $\mathrm{~mm}$ \\
\hline Material thickness & $h_{m a t}$ & 2 & $\mathrm{~mm}$ \\
\hline Applied field & $E_{0}$ & $7,8,9,10$ & $\mathrm{kVmm}^{-1}$ \\
\hline Initial surface charge density & $\rho_{s 0}$ & $1 \times 10^{-4}$ & $\mathrm{Cm}^{-2}$ \\
\hline Cavity surface conductivity & $\sigma_{s}$ & $1.394 \times 10^{-10} \exp \left(0.4108 E_{0}\right)$ & $\mathrm{Sm}^{-1}$ \\
\hline Inception field & $E_{i n c}$ & 3.41 & $\mathrm{kVmm}^{-1}$ \\
\hline Extinction field & $E_{\text {ext }}$ & 1.1 & $\mathrm{kVmm}^{-1}$ \\
\hline Initial EGR due to surface emission & $N_{e s 0}$ & $3000\left(N_{e s 0 H}\right), 150\left(N_{e s 0 L}\right)$ & $\mathrm{s}^{-1}$ \\
\hline $\begin{array}{l}\text { Electron generation rate due to } \\
\text { volume ionization }\end{array}$ & $N_{e v}$ & $98.19\left[1-\left(E_{0} / 5.874\right)^{-4.685}\right]$ & $\mathrm{s}^{-1}$ \\
\hline
\end{tabular}

significantly over the range of applied field used in the experiment.

The value for $\rho_{s 0}$ is set equal to $1 \times 10^{-4} \mathrm{Cm}^{-2}$ because if it is too large, $E_{\text {cav }}(t, 0,0)$ will rapidly reduce, resulting in discharges terminating significantly below $E_{\text {ext }}$ and resulting in a less precise PD charge magnitude. On the other hand, if it is set too small, the simulation time is greatly increased for little gain in simulation performance. Thus, $\rho_{s 0}$ was chosen to ensure a reasonable simulation time and good precision of PD charge magnitude, while ensuring that discharge curtails at an instant when the level is very close to $E_{\text {ext }}$.

$N_{e s 0 H}$ and $N_{e s 0 L}$ are constant because in the model the electron generation rate due to surface emission, $N_{e s}(t)$ is controlled by the field at the cavity centre, $E_{c a v}(t, 0,0)$, where higher applied fields cause higher maximum magnitudes of $E_{\text {cav }}(t, 0,0)$, resulting in an increased maximum of $N_{e s}(t)$. From sensitivity analysis, $N_{e v}$ is found to increase with the applied field, $E_{0}$. Higher $E_{0}$ may increase the likelihood of free electron generation rate in the cavity through volume ionization. For the range of applied stresses studied, from analysis, it is possible to express $N_{e v}$ as a function of $E_{0}$ using

$$
N_{e v}=\alpha\left[1-\left(\frac{E_{0}}{\beta}\right)^{-\gamma}\right]
$$

where $\alpha=98.19 \mathrm{~s}^{-1}, \beta=5.874 \mathrm{kVmm}^{-1}$ and $\gamma=4.685$ (for $7 \leq E_{0} \leq 10 \mathrm{kVmm}^{-1}$ ).

When the applied field is higher, surface charge moves faster along the cavity wall. Thus, charge moves a longer distance along the cavity wall per unit time, resulting in a shorter time is taken before charge recombination occurs on the cavity wall. However, in this model, the distance of the charge movement along the cavity wall per unit time for different applied field is assumed to be the same. The difference of the time for the first charge starts to recombine between different applied field is assumed to be negligible. Therefore, the rate of the amount of charge decay through charge recombination is determined by the field along the cavity wall and the surface conductivity. 
In order to match the simulation and measurement results of the maximum charge magnitude, $q_{\max }$, the surface conductivity, $\sigma_{s}$ is varied for each applied field, $E_{0}$. If $\sigma_{s}$ is set equal to zero, or lower or higher than a specific value, the simulated $q_{\max }$ will not match the measured data. From simulation data, analysis yields figure 9 and it is possible to express $\sigma_{s}$ as a function of $E_{0}$ using

$$
\sigma_{s}=\sigma_{s 0} \exp \left(\lambda E_{0}\right)
$$

where $\sigma_{s 0}=1.394 \times 10^{-10} \mathrm{Sm}^{-1}$ and $\lambda=0.4108 \mathrm{mmkV}^{-1}\left(\right.$ for $7 \leq E_{0} \leq 10 \mathrm{kVmm}^{-1}$ ). The exponential expression is similar to the one that has been obtained from previous work, where $\sigma_{s}$ increases with the applied field [18]. This corresponds to a faster charge movement along the cavity surface when the applied field is larger, resulting in greater amount of charge reduction with time. The cavity surface conductivity has been assumed to be the same for the whole cavity surface. In reality, the cavity surface that is directly exposed to PD activity may have higher conductivity than the region that is not directly exposed to PD [19].

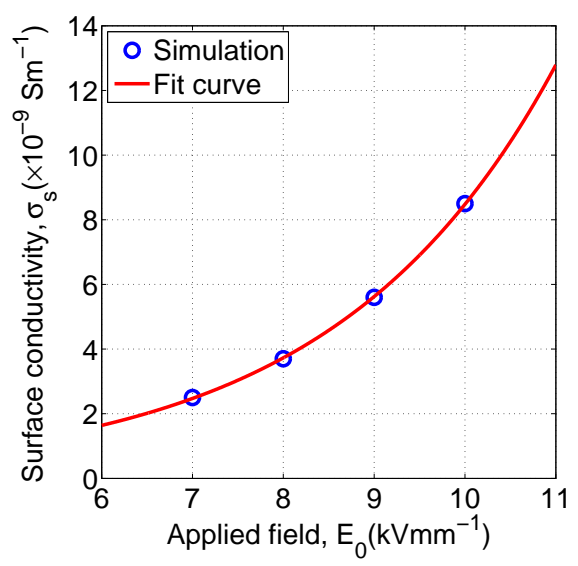

Figure 9. Calculated surface conductivity as a function of applied field magnitude (All simulation parameters are similar to those in figure 8).

Equations (18) and (19) are valid for different cavity radius, $r_{c a v}$. This is due to the field enhancement in the cavity, $f_{c}$ is independent of rcav for spherical cavity. Thus, the field in the cavity does not change with $r_{c a v}$, hence the expression is still a function of the applied field for different $r_{c a v}$. However, values in these expressions may be different. This is because $N_{e v}$ depends on cavity size, where $N_{e v}$ is higher in a larger cavity. For cavity surface conductivity, $\sigma_{s}$, the constant for equation (19) may also be different because different $r_{c a v}$ may have different surface conductivity. For a given applied voltage, the material thickness, $h_{\text {mat }}$ will determine the magnitude of the applied field. When the permittivity, $\varepsilon_{r}$ is changed, the field enhancement factor, $f_{c}$ in the cavity changes, thus the field in the cavity also changes. However, the field in the cavity is still proportional to the applied field, as it is equal to $f_{c} E_{0}$. Since the field in the cavity is a function of the applied field, $E_{0}$, equations (18) and (19) are valid for different $\varepsilon_{r}$. 
When $h_{\text {mat }}$ is increased, the applied field becomes lower. Thus, the number of PDs per cycle will reduce. This also causes the measured inception voltage to be higher because the field in the cavity is lower due to lower applied field. The apparent charge magnitude detected from the measurement will also be lower because the distance between the discharge source and the detecting electrode is further when the material thickness is increased. When $\varepsilon_{r}$ is increased, the field enhancement in the cavity increases. Thus, the number of PDs per cycle will become higher. This also causes the measured inception voltage to be lower because the field in the cavity is higher due to the higher applied field. The apparent charge magnitude detected from the measurement will be higher because of higher field enhancement in the cavity.

\subsection{PD behaviour as a function of spherical cavity size}

Table 8 shows the obtained measurement $(\mathrm{M})$ results for spherical cavities of radius $0.55 \mathrm{~mm}$ and $1.175 \mathrm{~mm}$ within a dielectric material of thickness $3 \mathrm{~mm}$ at $50 \mathrm{~Hz}, 6$ $\mathrm{kVmm}^{-1}$ ac sinusoidal applied field while figure 10 shows the corresponding $\phi-q-n$ plots of the measurement results. The number of PDs per cycle is higher but the total charge magnitude per cycle and the mean charge, maximum and minimum charge magnitudes are lower for the smaller cavity than the larger cavity. Since there may be more free charges accumulated along the larger cavity surface after a discharge, surface charge decay through conduction along the cavity wall could be more signifcant than the smaller cavity, resulting in a lower electron generation rate and less PDs per cycle. There is more inactive time observed from the measurements in the larger cavity size, where no $\mathrm{PD}$ occurs at all and this may be due to very low electron generation rate [15].

The total charge magnitude per cycle and maximum charge magnitude increase with cavity radius because the cavity radius determines the maximum propagation length of the avalanche parallel with the applied field and the size of avalanche head perpendicular to the applied field [3]. Thus, in a larger spherical cavity, the avalanche head can grow larger, resulting in a larger maximum PD charge magnitude and higher total charge per cycle.

For the $\phi-q-n$ plots of the measurement results, the patterns seems to have the same characteristics as has been found for the PD experiment as a function of the applied field. There are PDs occur near the minimum and higher charge mangitudes. The reasons are the same as what have been detailed for the experiment as a function of the applied field. The differences between the patterns obtained for the smaller and

larger cavity sizes are the distribution of the PD events occurring near the minimum and higher charge magnitudes. For the smaller cavity, the pattern of PD events near the minimum charge magnitude is clearly distinguished from the 'rabbit-ear' like pattern. The 'rabbit-ear' like pattern has a sharp end, which can be seen at 90 and 270 degrees near to the maximum charge magnitude. Due to higher electron generation rate which may be due to lower charge decay effect between consecutive discharges, the statistical time lag becomes shorter. Hence, more PDs occur with around the minimum charge 
Table 8. PD data of measurement (M) and simulation (S) results for two different cavity radii.

\begin{tabular}{lllll}
\hline Cavity radius, $r_{\text {cav }}(\mathrm{mm})$ & \multicolumn{3}{c}{0.55} & \multicolumn{2}{c}{1.175} \\
\cline { 2 - 5 } & $\mathrm{M}$ & $\mathrm{S}$ & $\mathrm{M}$ & $\mathrm{S}$ \\
\hline Total PDs per cycle & 6.5 & 6.5 & 2.5 & 2.5 \\
Total charge per cycle $(\mathrm{pC})$ & 651 & 664 & 5420 & 5469 \\
Mean charge (pC) & 101 & 102 & 2165 & 2184 \\
Maximum charge, $q_{\max }(\mathrm{pC})$ & 373 & 379 & 4763 & 4781 \\
Minimum charge, $q_{\min }(\mathrm{pC})$ & 80 & 80 & 938 & 938 \\
\hline
\end{tabular}

mangnitude and at earlier phase of the applied field.

However, from the measured $\phi-q-n$ plots for the larger cavity, there is no obvious separation between patterns of PD events occurring near the minimum and higher charge magnitudes. The 'rabbit-ear' like pattern does not have a sharp end like for the smaller cavity, but it seems to be dispersing. This may be due to a higher charge decay effect between consecutive discharges, reducing the electron generation rate, causing the statistical time lag becomes longer. Hence, more PDs occur at field level higher than the inception field, resulting in the number of PD events occurring with larger charge magnitude to be high.

The model geometry in this work can be also used for simulation of different spherical cavity radiuses. For this purpose, simulation models with cavity radiuses, $r_{\text {cav }}$ of $0.55 \mathrm{~mm}$ and $1.175 \mathrm{~mm}$ within a dielectric material of $3 \mathrm{~mm}$ thickness were developed. Results were compared for PD measurement data from samples with two different cavity radiuses. In order to match $q_{\text {real }}$ obtained from the FEA model and the $q_{\text {real }}$ calculated from equation (3) for the same $\Delta E_{\text {cav }}$, it has been found that for $r_{c a v}$ equals to $0.55 \mathrm{~mm}$, charge density, $\rho_{s}(t, r, z)$ is added on regions 1 to 6 of the upper and lower cavity surfaces during a discharge event but is added only on region 1 for $r_{c a v}$ equals to $1.175 \mathrm{~mm}$.

Comparison of $\phi-q-n$ plots between the measurement and simulation results for different cavity radiuses is shown in figure 10 and table 8 details their PD data. Table 9 shows the simulation parameters used in the model. Both are within reasonable agreement compared to each other. All parameters for the simulation $\left(E_{\text {inc }}, E_{\text {ext }}, \rho_{s 0}\right.$, $N_{e s 0 H}, N_{e s 0 L}, N_{e v}$ and $\sigma_{s}$ ) were determined using the same method that was used in section 5.2.

The inception field, $E_{i n c}$ is found to be higher for the smaller cavity, which is in agreement with the literature [3, 4]. Since the electric field enhancement in the cavity is not influenced by the spherical cavity radius, $E_{\text {inc }}$ only depends on the pressure in the cavity [20, 21]. The extinction field, $E_{\text {ext }}$ is found to be higher for the smaller cavity in order to reproduce the measured minimum charge magnitude. This follows that higher 

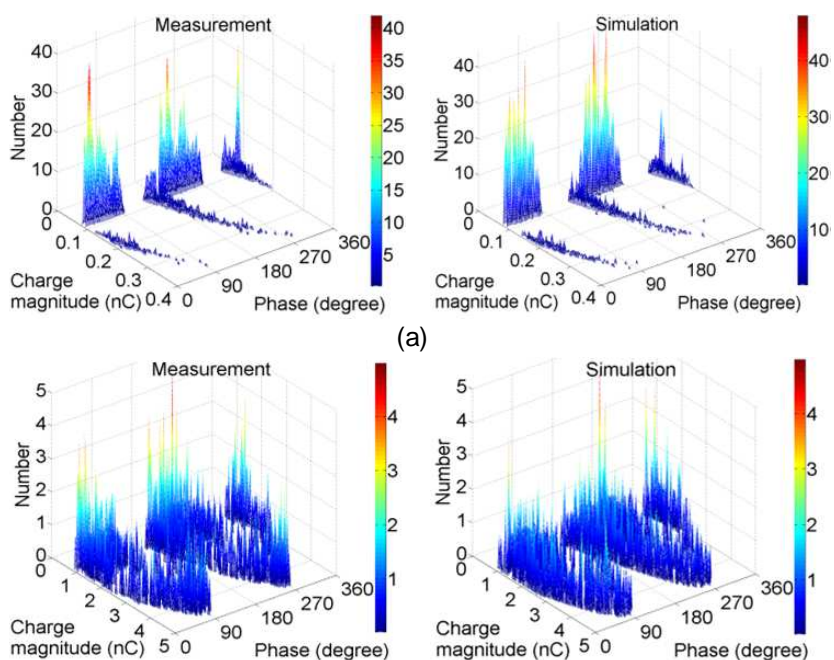

(b)

Figure 10. $\phi-q-n$ plots of measurement and simulation results for two different cavity radius: (a) $r_{\text {cav }}=0.55 \mathrm{~mm}$ and (b) $r_{c a v}=1.175 \mathrm{~mm}$.

Table 9. Simulation parameters used in the model for figure 10.

\begin{tabular}{lllll}
\hline Definition & Symbol & Value & Value & Unit \\
\hline Cavity radius & $r_{\text {cav }}$ & 0.55 & 1.175 & $\mathrm{~mm}$ \\
Material thickness & $h_{\text {mat }}$ & 3 & 3 & $\mathrm{~mm}$ \\
Applied field & $E_{0}$ & 6 & 6 & $\mathrm{kVmm}^{-1}$ \\
Initial surface charge density & $\rho_{s 0}$ & $2 \times 10^{-5}$ & $1.2 \times 10^{-4}$ & $\mathrm{Cm}^{-2}$ \\
Cavity surface conductivity & $\sigma_{s}$ & $5 \times 10^{-10}$ & $1.7 \times 10^{-8}$ & $\mathrm{Sm}^{-1}$ \\
Inception field & $E_{\text {inc }}$ & 3.4 & 2.8 & $\mathrm{kVmm}^{-1}$ \\
Extinction field & $E_{e x t}$ & 0.6 & 0.55 & $\mathrm{kVmm}^{-1}$ \\
Higher initial EGR due to surface emission & $N_{e s 0 H}$ & 10 & 0.25 & $\mathrm{ks}^{-1}$ \\
Lower initial EGR due to surface emission & $N_{e s 0 L}$ & 5 & 0.125 & $\mathrm{ks}^{-1}$ \\
Electron generation rate due to volume & $N_{e v}$ & 0.01 & 0.1 & $\mathrm{ks}^{-1}$ \\
ionization & & & & \\
\hline
\end{tabular}

$E_{\text {inc }}$ causes $E_{\text {ext }}$ to be higher as well.

For a larger cavity, longer time may be taken for charge to reach the point where the upper and lower cavity surface meets before charge recombination occurs. However, in this model, the time taken for charge to reach the end of the cavity wall for both smaller and larger cavities is assumed to be the same. This is possible because in the larger cavity, the charge density on each surface region is higher, resulting in charge to move faster along the cavity wall.

A higher surface conductivity, $\sigma_{s}$ is assigned for the larger cavity than the smaller 
cavity in order to match the measured maximum charge magnitude. Referring to the charge magnitude-phase axes of the $\phi-q$ - $n$ plots, for the smaller cavity, the tail of the 'rabbit-ear' like pattern is well-defined but for the larger cavity, it is more dispersed. This could be due to the effect of surface charge decay through surface conduction being more significant in the larger cavity, which results in higher $\sigma_{s}$ in the simulation. Since many PDs occur with large charge magnitude in the larger cavity, charge bombardment on the cavity surface is more rapid. This may cause faster chemical deterioration of the cavity wall, resulting in an increase of the surface conductivity.

$\rho_{s 0}$ is set higher for the larger cavity because the maximum propagation length of the avalanche parallel with the applied field and the avalanche head that is perpendicular to the applied field are both larger. These results infer that a higher amount of chargesare landing on the cavity surface and there is more charge propagation along the cavity wall. Consequently, this results in a higher surface charge density on the larger cavity surface [3].

The electron generation rate (EGR) due to surface emission, $N_{e s 0 H}$ and $N_{e s 0 L}$ are higher for the smaller cavity than the larger cavity. As has been obtained from the FEA model, the ratio of trapped charge density to the total charge density on the cavity surface after a PD occurs is higher for the smaller cavity than the larger cavity. Thus, this results in higher EGR due to surface emission. This is due to charges propagating in more regions of the cavity surface in the smaller cavity. However, $N_{e v}$ is higher for the larger cavity due to its larger volume, where the electron generation rate through volume ionization is higher in a larger cavity volume. $N_{e v}$ is independent of the surface charge distribution along the cavity wall.

\subsection{Sensitivity analysis on the simulation parameters}

As has been mentioned in the modelling of PD occurrence section, sensitivity analysis has been used to determine the values for parameters related to electron generation rate, i.e. $N_{e s 0 H}, N_{e s 0 L}$ and $N_{e v}$. An optimization method has been used for this purpose. Initially, $N_{e s 0 H}, N_{e s 0 L}$ and $N_{e v}$ were defined with small values. Then, $N_{e s 0 H}$ is increased until the global minimum from the MSE vs. $N_{e s 0 H}$ curve is identified. After that, the value for $N_{e s 0 L}$ is increased and the simulation is run again with initial value of $N_{e s 0 H}$. $N_{e s 0 H}$ is increased until the global minimum from the MSE vs. $N_{e s 0 H}$ curve is identified. The value for $N_{e s 0 L}$ is increased again and $N_{e s 0 H}$ is reinitialized. The simulation is run until the global minimum from the MSE vs. $N_{e s 0 L}$ curve is identified. Once it has been identified, the value for $N_{e v}$ is increased and the values for $N_{e s 0 L}$ and $N_{e s 0 H}$ are reinitialized. The whole process above is repeated and only stops once the MSE is seen to be keep increasing for different combination values of $N_{e s 0 H}, N_{e s 0 L}$ and $N_{e v}$.

Table 10 shows the numbers of combination values of $N_{e s 0 H}, N_{e s 0 L}$ and $N_{e v}$ for the simulation of PD as a function of applied field at $7 \mathrm{kVmm}^{-1}$ which has been performed in section 5.2. For each combination, the number of PDs per cycle and the MSE between the simulation and measurement results are calculated, as shown in Figures 11. This 
Table 10. Numbers of combination values of $N_{e s 0 H}, N_{e s 0 L}$ and $N_{e v}$.

\begin{tabular}{llll}
\hline $\begin{array}{l}\text { Combination } \\
\text { number }\end{array}$ & $N_{e v}$ & $N_{e s 0 L}$ & $N_{e s 0 H}$ \\
\hline 1 & 5 & 0 & 0 \\
2 & 5 & 0 & increases by 500 \\
3 & $\cdot$ & $\cdot$ & $\cdot$ \\
$\cdot$ & $\cdot$ & $\cdot$ & $\cdot$ \\
$\cdot$ & $\cdot$ & 0 & until 10000 \\
$\cdot$ & $\cdot$ & increases by 50 & for each $N_{e s 0 L}$, \\
$\cdot$ & $\cdot$ & $\cdot$ & increases $N_{e s 0 H}$ by 500 \\
$\cdot$ & 5 & $\cdot$ & until 10000 \\
$\cdot$ & increases by 5 & for each $N_{e v}$, increases & for each $N_{e v}$ and \\
$\cdot$ & $\cdot$ & $N_{e s 0 L}$ by 50 until 300 & $N_{e s 0 L}$, increases $N_{e s 0 H}$ \\
$\cdot$ & $\cdot$ & & by 500 until 10000 \\
$\cdot$ & until 100 & & \\
\hline 240 & & & \\
\hline
\end{tabular}

method was used for every applied field and both cavity sizes.

From the simulation of number of PDs per cycle versus combination number, a tolerance of \pm 0.1 from the measured number of PDs per cycle (2.5) is taken, as shown in figure 11(a). The MSE of all combinations that fall within this range are evaluated in 11(b), where the least MSE or global minimum is identified. It was found that the global minimum of MSE was found in the of combination range between 1520 to 1570 , which falls within the acceptable range of the number of PDs per cycle and reproduces the $\phi-q-n$ plot of the measurement results in figure 8 for $7 \mathrm{kVmm}^{-1}$. Thus, the values of $N_{e s 0 H}, N_{e s 0 L}$ and $N_{e v}$ for combinations between 1520 and 1570 which yields the least MSE are selected as the simulation parameter values. It was therefore found that $N_{e s 0 H}$ $=3000, N_{e s 0 L}=150$ and $N_{e v}=55$ yield the best simulation results compares to the measurement results.

Figure 12 shows $\phi-q-n$ plots of the simulation using different values of $N_{e s 0 H}, N_{e s 0 L}$ and $N_{e v}$ that yield the number of PDs per cycle within the acceptance criteria. However, it can be seen that there is significant variation in these plots when different combinations of parameter values are used. Refering to $\phi-q-n$ plots in figure 12 , PD patterns near the minimum charge mangitude are determined by $N_{e s 0 H}$ while the 'rabbit-ear' like curves are dependent on $N_{e s 0 L}$ and $N_{e v}$. A higher $N_{e s 0 H}$ causes the number of PDs per cycle to occur with minimum charge magnitude to increase (figure $12(\mathrm{~b})$ ) while a lower value causes less PDs with minimum charge magnitude (figure 12(d)). Therefore, different combination values of these simulation parameters will yield very different PD patterns. 


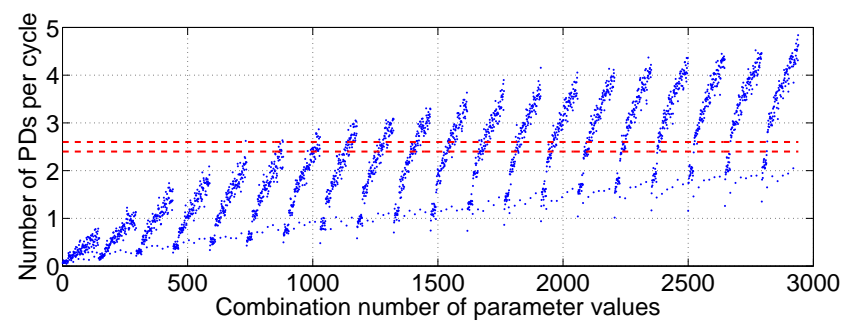

(a)

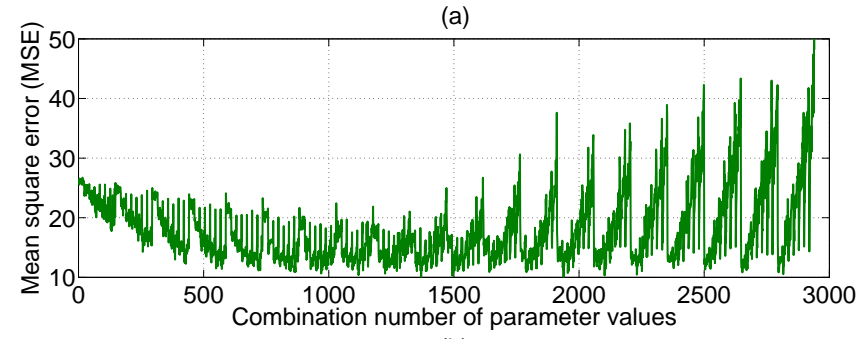

(b)

Figure 11. Results from the sensitivity analysis performed to select the best values for $N_{e s 0 H}, N_{e s 0 L}$ and $N_{e v}$ (all parameters unchanged).

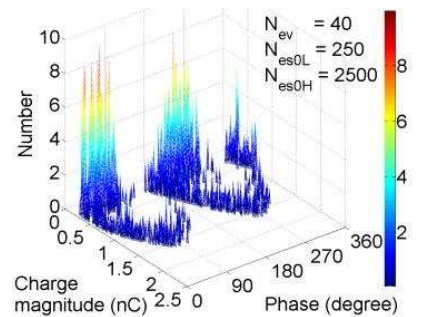

(a)

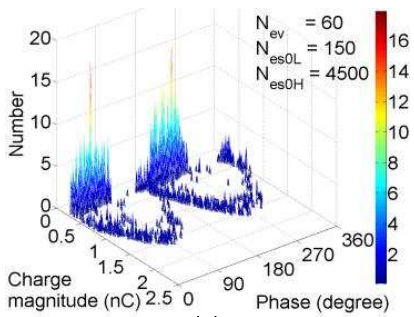

(c)

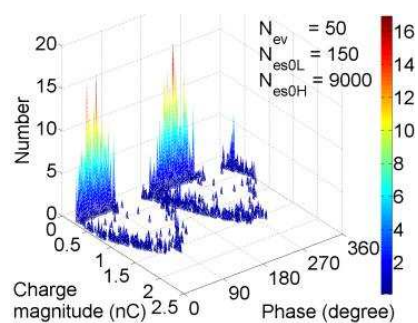

(b)

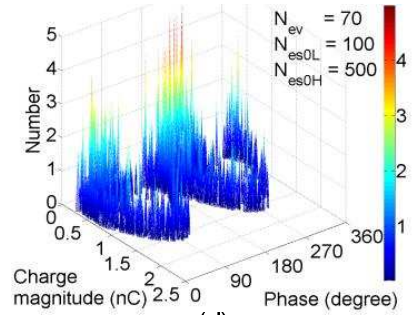

(d)

Figure 12. $\phi-q-n$ plots of the simulation using different values of $N_{e s 0 H}, N_{e s 0 L}$ and $N_{e v}$.

\section{Conclusion}

A model that allows simulation of PD for a single void within a dielectric material has been developed. The influence of cavity surface charge distribution on the electric field distribution in the cavity and the material has been studied using Finite Element Analysis method. The cavity surface charge distribution is determined by charge propagation on the cavity surface during a PD event and charge movement along the cavity wall under the influence of the electric fields polarity and magnitude. This consequently affects the likelihood of subsequent discharges occurring. The surface charge density, inception field, extinction field and surface conductivity are the 
main parameters that control the cavity surface charge distribution. Comparison of measurement and simulation results as a function of the applied field and spherical cavity size have been undertaken to validate the simulation model. It has been found that the surface conductivity increases with the applied field and cavity size, where surface charge moves faster along the cavity wall, resulting in higher charge decay rate through charge recombination and faster decrement of the electric field due to the surface charge. The PD repetition rate in the cavity has been identified to be mainly dependent on the electron generation rate due to surface emission and volume ionization.

\section{References}

[1] Rainer P and Farhad B 2002 J. Phys. D: Appl. Phys. 35 25-32

[2] Benzerouk D, Menzel J and Patsch R 2007 IEEE Int. Conf. on Solid Dielectrics p 552-555

[3] Niemeyer L 1995 IEEE Trans. Dielectr. Electr. Insul. 2 510-528

[4] Gutfleisch F and Niemeyer L 1995 IEEE Trans. Dielectr. Electr. Insul. 2 729-743

[5] Schifani R, Candela R and Romano P 2001 IEEE Trans. Dielectr. Electr. Insul. 8 589-597

[6] Kai W, Suzuoki Y, Mizutani T and Hengkun X 1999 IEEE Trans. Dielectr. Electr. Insul. 6 181-190

[7] Serdyuk Y V and Gubanski S M 2005 IEEE Trans. Dielectr. Electr. Insul. 12 725-735

[8] Kai W, Yasuo S and Dissado L A 2004 J. Phys. D: Appl. Phys. 37 1815-1823

[9] Kai W, Okamoto T and Suzuoki Y 2007 IEEE Trans. Dielectr. Electr. Insul. 14 461-470

[10] Ahmed N and Srinivas N 2001 IEEE Conf. on Electrical Insulation and Dielectric Phenomena p $307-310$

[11] Forssen C and Edin H 2008 IEEE Trans. Dielectr. Electr. Insul. 15 1610-1616

[12] Edin H PhD Thesis 2001

[13] Kindersberger J and Lederle C 2008 IEEE Trans. Dielectr. Electr. Insul. 15 941-948

[14] Illias H A, Chen G and Lewin P L 2009 Int. Symp. on High Voltage Engineering p 1-6

[15] Illias H A, Chen G and Lewin P L 2011 IEEE Electr. Insul. Mag. 27 38-45

[16] Illias H A, Chen G and Lewin P L 2011 IEEE Trans. Dielectr. Electr. Insul. 18 432-443

[17] Illias H A, Chen G and Lewin P L 2010 IEEE Int. Conf. on Solid Dielectrics p 561-564

[18] Weedy B M 1985 IEE Proc. A Physical Science, Measurement and Instrumentation 132 450-454

[19] Hudon C, Bartnikas R and Wertheimer M R 1990 IEEE Int. Symp. on Electrical Insulation p 153-155

[20] Crichton G C, Karlsson P W and Pedersen A 1989 IEEE Trans. Electr. Insul. 24 335-342

[21] Pedersen A, Crichton G C and McAllister I W 1994 IEEE Conf. on Electrical Insulation and Dielectric Phenomena p 79-84 